\title{
Poking neurons and nerves precisely
}

\author{
The integration and miniaturization of components in electronic and photonic devices for interfacing with neural \\ tissue allow for ever more precise neural recording and stimulation.
}

M aking sense of the flow of information across large networks of neurons and along nerve fibres, and controllably modulating it, would open up enticing opportunities and help address long-sought challenges: deciphering how information is processed in the brain, interfering with the misregulation of signal flows underpinning epilepsy and other neurological disorders, restoring vision (partially) to the blind and making braincomputer interfaces that enable people to intuitively control and sense prostheses, wearables or external devices.

Although these prospects will remain as science fiction for the foreseeable future, devices that can simultaneously and precisely 'poke' hundreds or thousands of individual neurons, or that can interface with nerve fibres in the body via less invasive means, are enabling the capture of more information from neural tissue than has ever been possible, as well as the controlled stimulation of the tissue to either investigate the responses, to restore function or to augment it. These devices are all benefiting from the progressive miniaturization, integration and largescale fabrication of electronic and photonic components. This brings about challenges (Nat. Biomed. Eng. 1, 0053; 2017) related to the in-device power storage, to wireless power transmission through tissue, and to materials, device designs and fabrication strategies (Nat. Biomed. Eng. 3, 1-2; 2019) that improve device biocompatibility and durability.

Five papers included in this issue showcase what is possible today, and some of the challenges ahead. First, to understand the connectivity of neurons through the flow of action potentials, it is necessary to measure subthreshold events such as postsynaptic potentials (that is, potentials that can excite or inhibit action potentials). Arrays of patch-clamp electrodes can be used to interrogate neurons in culture, yet they are not scalable (only tens of neurons have been measured in parallel with patch-clamp arrays). And existing multielectrode arrays are typically 'blind' to neurons that do not fire and do not measure multiple types of synaptic potentials simultaneously. Donhee Ham, Hongkun Park and colleagues show in an Article that subthreshold potentials and action

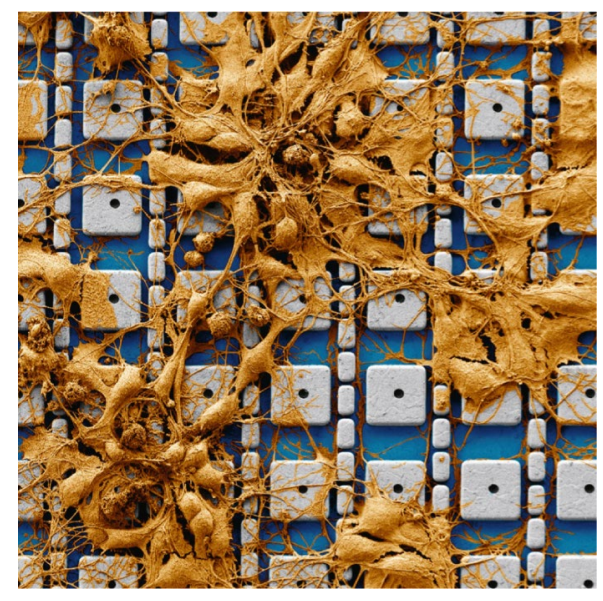

Credit: Figure reproduced from Abbott, J. et al. Nat. Biomed. Eng. https://doi.org/10.1038/s41551019-0455-7 (2019), Springer Nature Ltd

potentials can be recorded (and action potentials stimulated) simultaneously across thousands of connected neurons in vitro via an array of vertical nanoelectrodes (Fig. 1) that electroporate the neurons (so as to gain intracellular access to record postsynaptic potentials). The device, fabricated on a silicon chip that integrates low-noise voltage amplifiers, can accurately determine the synaptic connectivity of thousands of neurons with hundreds of synaptic connections. Yet, as noted by Micha Spira in an accompanying News \& Views, "a significant increase in the stability and durability of the intracellular recording configuration from minutes to months would be needed to enable the analysis of the electrophysiological signals underlying different forms of network plasticity (learning and memory), neuropathological processes and the long-term effects of drugs and toxins."

In another Article, Michal Lipson, Adam Kepecs and colleagues report a nanophotonic silicon probe based on complementary metal-oxide-semiconductor technology (the most common technology for the fabrication of integrated circuits) implanted in the brain of anaesthetized mice that can stimulate spike patterns in small groups of neurons in the animal's brain at sub-millisecond precision by switching multiple optical beams in less than $20 \mu \mathrm{s}$.
By integrating electrical recording sites into the device, the researchers also show that optical stimulation and electrical recording of neural activity can be performed simultaneously. State-of-the-art implantable silicon probes had mostly used passive nanophotonic probes relying on external table-top optics (thus limiting the number of optical stimulation sites and impairing chronic implantability) or micrometrescale light-emitting diodes, which come with larger illumination volumes (and thus resolutions larger than $100 \mu \mathrm{m}$ ) and with switching frequencies in the range of tens of Hertzs. Reductions in the overall footprint of the tip of the implantable device (which is nearly 1-mm wide as reported) will facilitate experiments in freely behaving animals.

Within the retina, activated photoreceptors pass a neuronal signal through to ganglion cells (via relay neurons such as bipolar cells). The axons of the ganglion cells leave the retina via the optic nerve, which carries the sensory information (from more than a million ganglion cells) to primary visual centres in the brain. Retinal prostheses intended for the blind can bypass damaged photoreceptors and deliver electrical stimuli to the ganglion cells. In a third Article, Serge Picaud and colleagues report the evaluation of an existing miniaturized photovoltaic subretinal prosthesis sensitive to near-infrared light (generated by an external image-projection system) in awake macaques. The passive photovoltaic implant, which can activate the primate retina with light spots (known as phosphenes) $200-250 \mu \mathrm{m}$ in diameter, triggered behavioural responses in the monkeys for at least two years after device implantation. Further miniaturization of the wireless prosthesis should allow for increases in resolution and visual acuity.

Retinal prostheses are however unsuitable for retinas severely damaged as a result of retinal detachment or traumatic eye injury, for example. In such cases, the optic nerve can be stimulated directly. A fourth Article, by Diego Ghezzi and colleagues, show that a modified version of a previously described intraneural electrode array can selectively activate optic-nerve fibres in rabbits. To activate fibres close to the centre of the optic nerve (which is challenging to achieve with cuff electrodes) and to minimize damage to it when the device is inserted, the electrode 
array features cut-out flaps that only bend outwards on slight retraction of the device after its insertion. Integrating more electrodes into the array would increase the density of phosphenes and thus the provided visual aid.

Implanted electrical stimulators that can restore some degree of motor control by providing somatosensory input into severed nerves suffer from a range of limitations: in particular, they are too large (thousands of cubic millimetres) and thus too invasive, long-term function (often needed for decades) is not guaranteed and, most often, they require transcutaneous wires, which are a source of infection and device failure.
In a fifth Article, Rikki Muller, Michel Maharbiz, Jose Carmena and colleagues describe a fully implantable $1.7 \mathrm{~mm}^{3}$ wireless and battery-free neural stimulator that integrates, on a nerve cuff, stimulation electrodes, a piezoceramic ultrasonic transducer, an energy-storage capacitor and an integrated circuit for power rectification, communication and stimulation. The researchers tested the device on the sciatic nerve of rats, showing that it allows for repeatable stimulation across a range of physiological responses. Being ultrasonically powered, the device can function at much larger depths than electromagnetically powered implants. Still, achieving selective (rather than blunt) stimulation, as noted by Max Ortiz-Catalan in an accompanying News \& Views, will require further miniaturization and integration efforts.

Effectively interfacing devices with human neural tissue demands device biocompatibility and robustness, sufficient signal resolution, selectivity and sensitivity, as well as implant durability and removability. All of these challenges can benefit from component integration and device miniaturization.

Published online: 19 February 2020 https://doi.org/10.1038/s41551-020-0523-Z 